Cahiers $d u$ MONDE RUSSE

\section{Cahiers du monde russe}

Russie - Empire russe - Union soviétique et États indépendants

$55 / 3-4 \mid 2014$

Varia

\title{
Vladislav Rjeoutski, Alexandre Tchoudinov, éds. Le précepteur francophone en Europe : $\mathrm{XVII}^{\mathrm{e}}$-XIX ${ }^{\mathrm{e}}$ siècles
}

\section{Nikolaj Promyslov}

\section{OpenEdition}

\section{Journals}

Édition électronique

URL : http://journals.openedition.org/monderusse/8037

DOI : $10.4000 /$ monderusse. 8037

ISSN : $1777-5388$

\section{Éditeur}

Éditions de l'EHESS

\section{Édition imprimée}

Date de publication : 1 juillet 2014

Pagination : 366-370

ISBN : 978-2-7132-2441-6

ISSN : $1252-6576$

\section{Référence électronique}

Nikolaj Promyslov, « Vladislav Rjeoutski, Alexandre Tchoudinov, éds. Le précepteur francophone en Europe : xvII -хІх siècles », Cahiers du monde russe [Онлайн], 55/3-4 | 2014, Выложить онлайн 10 avril 2015, Наводить справки в 23 septembre 2020. URL : http://journals.openedition.org/monderusse/ 8037 ; DOI : https://doi.org/10.4000/monderusse.8037

Ce document a été généré automatiquement le 23 septembre 2020.

(c) École des hautes études en sciences sociales 


\title{
Vladislav Rjeoutski, Alexandre Tchoudinov, éds. Le précepteur francophone en Europe : XVII ${ }^{\mathrm{e}}-\mathrm{XIX}^{\mathrm{e}}$ siècles
}

\author{
Nikolaj Promyslov
}

\section{RÉFÉRENCE}

Vladislav Rjeoutski, Alexandre Tchoudinov, éds., Le précepteur francophone en Europe : XVII ${ }^{\mathrm{e}}$-XIX ${ }^{\mathrm{e}}$ siècles, L'Harmattan, 2013, $462 \mathrm{p}$.

1 В ЕВРОПЕЙСКОЙ КУЛЬТУРЕ НОВОГО ВРЕМЕНИ И ОСОБЕННО ЭПОХИ ПРОСВЕЩЕНИЯ ФРАНКОЯЗЫЧНЫЙ ГУВЕРНЕР БЫЛ ВАЖНЕЙШИМ ЛИЦОМ. СРЕДИ ГУВЕРНЕРОВ БЫЛО НЕМАЛО ИНТЕЛЛЕКТУАЛОВ И ДАЖЕ УЧЕНЫХ МИРОВОГО УРОВНЯ, НЕРЕДКО ПОПАДАЛИСЬ И АВАНТЮРИСТЫ, НО ВСЕ ОНИ ВОПЛОЩАЛИ В СЕБЕ ХАРАКТЕРНЫЕ ЧЕРТЫ ЭПОХИ, ВЫСТУПАЯ ПОСЛАННИКАМИ « РЕСПУБЛИКИ УЧЕНЫХ». ОСОБОЕ ЗНАЧЕНИЕ ЭТА ФИГУРА ИМЕЛА ДЛЯ РОССИИ ХVIII-ХIX ВВ., ГДЕ ВОСПИТАНИЕМ ДВОРЯНСКОЙ МОЛОДЕЖИ - БУДУЩЕЙ ЭЛИТЫ ГОСУДАРСТВА - ЗАНИМАЛИСЬ ПРЕИМУЩЕСТВЕННО ИНОСТРАНЦЫ. ВМЕСТЕ С ТЕМ В ХУДОЖЕСТВЕННОЙ ЛИТЕРАТУРЕ XVIII-XIX ВВ. ОЧЕНЬ ЧАСТО МОЖНО ВСТРЕТИТЬ НЕГАТИВНЫЙ ОБРАЗ ГУВЕРНЕРОВ. ЛИТЕРАТОРЫ СТАВИЛИ ПОД СОМНЕНИЕ ПОЛЕЗНОСТЬ ДЕЯТЕЛЬНОСТИ ФРАНЦУЗСКИХ УЧИТЕЛЕЙ ИЗ-ЗА НЕЗНАНИЯ ИМИ МЕСТНОЙ КУЛЬТУРЫ. В ДАЛЬНЕЙШЕМ МНОГИЕ ИСТОРИКИ ПРИНЯЛИ НА ВЕРУ УТВЕРЖДЕНИЯ ЛИТЕРАТОРОВ. ТАКОЙ НЕГАТИВНЫЙ ВЗГЛЯД НА ПРАКТИКУ ПРИГЛАШЕНИЯ ИНОСТРАННЫХ ГУВЕРНЕРОВ БЫЛ ХАРАКТЕРЕН ДЛЯ РЯДА ЕВРОПЕЙСКИХ ГОСУДАРСТВ В ПЕРИОД СТАНОВЛЕНИЯ НАЦИОНАЛЬНОГО САМОСОЗНАНИЯ ИХ НАРОДОВ.

СОСТАВИТЕЛИ РЕЦЕНЗИРУЕМОЙ КНИГИ ПОПЫТАЛИСЬ РАЗОБРАТЬСЯ, НАСКОЛЬКО ОБОСНОВАННЫМИ ИЛИ, НАПРОТИВ, НАДУМАННЫМИ БЫЛИ ЭТИ ДАВНИЕ СТЕРЕОТИПЫ И 
КАКОВА В ДЕЙСТВИТЕЛЬНОСТИ БЫЛА РОЛЬ ФРАНЦУЗСКИХ НАСТАВНИКОВ В ОБРАЗОВАНИИ, ВОСПИТАНИИ И В КУЛЬТУРНОЙ ЖИЗНИ ЕВРОПЫ.

ПРЕДСТАВЛЕННЫЙ ТРУД СОСТОИТ ИЗ ТРЕХ РАЗДЕЛОВ. ПЕРВЫЙ ПОСВЯЩЕН ИЗУЧЕНИЮ РОЛИ ФРАНКОЯЗЫЧНЫХ ГУВЕРНЕРОВ В ОБРАЗОВАТЕЛЬНОМ ПРОЦЕССЕ В СТРАНАХ ЦЕНТРАЛЬНОЙ И ЗАПАДНОЙ ЕВРОПЫ, ВТОРОЙ - РАБОТЕ ФРАНКОЯЗЫЧНЫХ ГУВЕРНЕРОВ В РОССИЙСКОЙ ИМПЕРИИ, А ТРЕТИЙ - ОТДЕЛЬНЫМ ПЕРСОНАЖАМ, СЛУЖИВШИМ ГУВЕРНЕРАМИ ИЛИ ПРЕПОДАВАТЕЛЯМИ В РАЗНЫХ СТРАНАХ.

ГЛАВЫ ПЕРВОГО РАЗДЕЛА ОСВЕЩАЮТ РАБОТУ ФРАНКОЯЗЫЧНЫХ ГУВЕРНЕРОВ В ЧЕХИИ, ПОЛЬШЕ, БРИТАНИИ И ИТАЛИИ. ПОДБОР СТРАН ОПРЕДЕЛЯЛСЯ ТЕМ, ЧТО ИМЕННО ЗДЕСЬ УВЛЕЧЕНИЕ ВСЕМ ФРАНЦУЗСКИМ НАЧАЛО РАЗВИВАТЬСЯ РАНЬШЕ И ПРИОБРЕЛО НАИБОЛЕЕ ШИРОКИЙ РАЗМАХ. В ЧЕХИИ, КАК ПОКАЗЫВАЕТ СТАТЬЯ И.СЕРМАН, ВЛИЯНИЕ ФРАНЦУЗОВ-ГУВЕРНЕРОВ НА СИСТЕМУ ОБРАЗОВАНИЯ СТРАНЫ БЫЛО, ВИДИМО, НЕСКОЛЬКО МЕНЬШЕ. КОЛИЧЕСТВО УЧИТЕЛЕЙ-ФРАНЦУЗОВ НИКОГДА НЕ ПРЕВЫШАЛО ЗДЕСЬ КОЛИЧЕСТВО УЧИТЕЛЕЙ, УРОЖЕНЦЕВ САМОЙ ЧЕХИИ. И ДАЖЕ ФРАНЦУЗСКИЙ ЯЗЫК В ЧЕХИИ ДАЛЕКО НЕ ВСЕГДА ПРЕПОДАВАЛИ ФРАНЦУЗЫ [С. 26]. В СТАТЬЕ М. КАМЕЦКОЙ ДЕМОНСТРИРУЕТСЯ ОБЩАЯ ДЛЯ ЕВРОПЫ ТЕНДЕНЦИЯ УТРАТЫ ПОПУЛЯРНОСТИ ЛАТИНСКОГО ЯЗЫКА И ПОСТЕПЕННОГО ПЕРЕХОДА НА ИЗУЧЕНИЕ ФРАНЦУЗСКОГО, ЧТО В ПОЛЬШЕ ПРОИСХОДИЛО БЛИЖЕ К СЕРЕДИНЕ ХVIII СТОЛЕТИЯ. С. ЛАХЕНИХТ РАССМАТРИВАЕТ СУДЬБУ ФРАНЦУЗСКИХ ГУГЕНОТОВ НА БРИТАНСКИХ ОСТРОВАХ И ИХ ВКЛАД В РАЗВИТИЕ ОБРАЗОВАНИЯ В СТРАНЕ. ВО МНОГИХ СТРАНАХ ЕВРОПЫ В XVII-XVIII ВВ. ГУГЕНОТЫ ВЫПОЛНЯЛИ РОЛЬ УЧИТЕЛЕЙ, НО В АНГЛИИ И ШОТЛАНДИИ ЭТОТ ПРОЦЕСС НАЧАЛСЯ ЕЩЕ В СЕРЕДИНЕ XVI СТОЛЕТИЯ. АВТОР ПОДЧЕРКИВАЕТ, ЧТО ГУГЕНОТЫ ПРЕПОДАВАЛИ РОДНОЙ ЯЗЫК НЕ ТОЛЬКО ИЗ СООБРАЖЕНИЙ МАТЕРИАЛЬНЫХ ВЫГОД, НО И ИЗ-ЗА ОЩУЩЕНИЯ ПРЕВОСХОДСТВА ФРАНЦУЗСКОГО ЯЗЫКА НАД ДРУГИМИ, ПОСКОЛЬКУ ОН СТАЛ УНИВЕРСАЛЬНЫМ ЯЗЫКОМ ОБЩЕНИЯ ДЛЯ ДИПЛОМАТОВ, ВОЕННЫХ И КОММЕРСАНТОВ [С. 62-63]. В СТАТЬЕ Н. МИНЕРВЫ, ПОСВЯЩЕННОЙ СУДЬБЕ ФРАНЦУЗСКИХ ПРЕПОДАВАТЕЛЕЙ В ИТАЛИИ, ПОДРОБНО РАССМАТРИВАЮТСЯ МЕТОДИКИ ПРЕПОДАВАНИЯ, А ТАКЖЕ УЧЕБНЫЕ ПОСОБИЯ, КОТОРЫЕ ИСПОЛЬЗОВАЛИ ГУВЕРНЕРЫ. В ПРИЛОЖЕНИИ К СТАТЬЕ ПЕРЕЧИСЛЕНЫ НАИБОЛЕЕ ЧАСТО ИСПОЛЬЗОВАВШИЕСЯ УЧЕБНИКИ [С. 83-87]. ЗАВЕРШАЕТСЯ РАЗДЕЛ СТАТЬЕЙ Ж.А. КАРАВОЛА О РОЛИ ГУВЕРНЕРОВ В РАЗВИТИИ МЕТОДИКИ ПРЕПОДАВАНИЯ НАЦИОНАЛЬНЫХ ЯЗЫКОВ В РАЗНЫХ СТРАНАХ; АВТОР ТАКЖЕ ПОДЧЕРКИВАЕТ РОЛЬ ГУГЕНОТОВ-ЭМИГРАНТОВ В ФОРМИРОВАНИИ НОВЫХ УЧЕБНЫХ СТАНДАРТОВ. ОТСУТСТВИЕ ОТДЕЛЬНОГО ТЕКСТА ПО ГЕРМАНИИ КОМПЕНСИРУЕТСЯ ТЕМ, ЧТО ВОПРОСЫ ПРЕПОДАВАНИЯ ФРАНЦУЗСКОГО ЯЗЫКА В ГЕРМАНИИ ОТЧАСТИ ЗАТРОНУТЫ В СТАТЬЕ Ж.А. КАРАВОЛА, А ТАКЖЕ В НЕКОТОРЫХ ГЛАВАХ ПОСЛЕДНЕГО РАЗДЕЛА КНИГИ.

5 РАЗДЕЛ О СУДЬБЕ ФРАНКОЯЗЫЧНЫХ ГУВЕРНЕРОВ В РОССИИ ОТКРЫВАЕТСЯ ОБОБЩАЮЩЕЙ ГЛАВОЙ В. РЖЕУЦКОГО, КОТОРАЯ ЗНАКОМИТ ЧИТАТЕЛЯ С НЕКОТОРЫМИ ИТОГАМИ МНОГОЛЕТНЕГО ПРОЕКТА, ПОСВЯЩЕННОГО ВСЕЙ ФРАНКОЯЗЫЧНОЙ ДИАСПОРЕ В РОССИИ В XVIII В., МОНУМЕНТАЛЬНЫМ РЕЗУЛЬТАТОМ КОТОРОГО СТАЛ ДВУХТОМНЫЙ СЛОВАРЬ, ОПУБЛИКОВАННЫЙ В $201 \Gamma^{1}{ }^{1}$ ОСТАЛЬНЫЕ ГЛАВЫ РАЗДЕЛА ЗНАКОМЯТ ЧИТАТЕЛЯ С НЕКОТОРЫМИ БОЛЕЕ УЗКИМИ ТЕМАМИ. В. БЕРЕЛОВИЧ ПОДРОБНО РАССМОТРЕЛ РОЛЬ ГУВЕРНЕРОВ ПРИ ПОДГОТОВКЕ И ПРОВЕДЕНИИ Grand Tour МОЛОДЫХ ГОЛИЦЫНЫХ ПО ЕВРОПЕ, А ТАКЖЕ ОЖИДАНИЯ И ТРЕБОВАНИЯ РОДИТЕЛЕЙ ПО ОТНОШЕНИЮ К ТАКОМУ ПУТЕШЕСТВИЮ, ЧТО ПРОЛИВАЕТ СВЕТ НА ПРЕДСТАВЛЕНИЯ 
ВЫСШЕЙ РОССИЙСКОЙ АРИСТОКРАТИИ ОБ ИДЕАЛЬНОМ ОБРАЗОВАНИИ ДЛЯ СВОИХ ДЕТЕЙ. СУДЬБЕ ФРАНЦУЗСКИХ ПРЕПОДАВАТЕЛЕЙ В МОСКВЕ В ПЕРВОЙ ПОЛОВИНЕ ХІХ В. ПОСВЯЩЕНА ГЛАВА О. СОЛОДЯНКИНОЙ. В ТОТ ПЕРИОД МОНАРХИЯ НАЧАЛА АКТИВНО СТРОИТЬ ГОСУДАРСТВЕННУЮ ОБРАЗОВАТЕЛЬНУЮ СИСТЕМУ И ПОТОМУ ПЫТАЛАСЬ УСТАНОВИТЬ КОНТРОЛЬ ЗА ЧАСТНЫМИ УЧИТЕЛЯМИ (И В ПЕРВУЮ ОЧЕРЕДЬ ИНОСТРАНЦАМИ), ЧТОБЫ, С ОДНОЙ СТОРОНЫ, НЕ ДОПУСТИТЬ РАСПРОСТРАНЕНИЯ ВОЛЬНОДУМСТВА, А С ДРУГОЙ, - ПОВЫСИТЬ КАЧЕСТВО ПРЕПОДАВАНИЯ В СТРАНЕ. ОСОБЫЙ ИНТЕРЕС ГЛАВЕ ПРИДАЮТ ТАБЛИЦЫ, НАГЛЯДНО ИЛЛЮСТРИРУЮЩИЕ НЕКОТОРЫЕ ВЫВОДЫ АВТОРА [С. 163-166]. ОТВЕТОМ НА РЕГЛАМЕНТАЦИЮ ЧАСТНОГО ОБРАЗОВАНИЯ СО СТОРОНЫ ГОСУДАРСТВА СТАЛО СОЗДАНИЕ ЧАСТНЫХ ШКОЛ. ПОДРОБНЕЕ ОБ ЭТОМ ПРОЦЕССЕ И УЧАСТИИ В НЕМ ФРАНКОЯЗЫЧНЫХ ГУВЕРНЕРОВ ГОВОРИТСЯ В ГЛАВЕ С. ФРОЛОВОЙ. ОСНОВНЫМ РЕГИОНОМ ИССЛЕДОВАНИЯ ДЛЯ АВТОРА СТАЛ КАЗАНСКИЙ УЧЕБНЫЙ ОКРУГ, ХОТЯ ИЗ ПРИВОДИМЫХ ПРИМЕРОВ ВИДНО, ЧТО ПРОЦЕССЫ СОЗДАНИЯ ЧАСТНЫХ УЧЕБНЫХ ЗАВЕДЕНИЙ БЫЛИ СХОДНЫ ВО МНОГИХ ГУБЕРНИЯХ РОССИЙСКОЙ ИМПЕРИИ. СОЗДАНИЕ И РАЗВИТИЕ ОБРАЗОВАТЕЛЬНОЙ СИСТЕМЫ В ОДЕССЕ И ВЛИЯНИЕ ФРАНЦУЗСКИХ УЧИТЕЛЕЙ НА ПЕРВЫЕ УЧЕБНЫЕ ЗАВЕДЕНИЯ В ГОРОДЕ РАССМОТРЕНЫ В СТАТЬЕ Е. ПОЛЕВЩИКОВОЙ, ПРОДОЛЖАЮЩЕЙ НЕБОЛЬШОЙ БЛОК РЕГИОНАЛЬНЫХ ИССЛЕДОВАНИЙ. ЗАРОЖДЕНИЕ СИСТЕМЫ ОБРАЗОВАНИЯ В ОДЕССЕ ВО МНОГОМ СВЯЗАНО С ФИГУРОЙ ГЕРЦОГА РИШЕЛЬЕ, МНОГО СИЛ И ВНИМАНИЯ УДЕЛЯВШЕГО МЕСТНЫМ УЧЕБНЫМ ЗАВЕДЕНИЯМ И ПРИВЛЕКАВШЕГО В ГОРОД КВАЛИФИЦИРОВАННЫХ ИНОСТРАННЫХ ПРЕПОДАВАТЕЛЕЙ. В ГЛАВЕ А. ЗЛАТОПОЛЬСКОЙ ПРОСЛЕЖИВАЕТСЯ ВЛИЯНИЕ ИДЕЙ Ж.Ж. РУССО НА СИСТЕМУ ПРЕПОДАВАНИЯ ДЕТЯМ ВЫСШЕЙ АРИСТОКРАТИИ. АВТОР ОБРАЩАЕТ ВНИМАНИЕ НА ТО, ЧТО, ХОТЯ В ЦЕЛОМ ТЕОРИЯ РУССО ОТНОСИТЕЛЬНО ОБРАЗОВАНИЯ СКОРЕЕ ОТВЕРГАЛАСЬ РУССКИМ ОБЩЕСТВОМ И ОСОБЕННО ЕКАТЕРИНОЙ II, ОТДЕЛЬНЫЕ ИДЕИ ФИЛОСОФА БЫЛИ ВЗЯТЫ НА ВООРУЖЕНИЕ ГУВЕРНЕРАМИ, УЧИТЕЛЯМИ И ОРГАНИЗАТОРАМИ УЧЕБНЫХ ЗАВЕДЕНИЙ ИМПЕРИИ. ОБРАЗ ГУВЕРНЕРА, ВЕДУЩЕГО ОБУЧЕНИЕ ПО СИСТЕМЕ РУССО, ИССЛЕДУЕТСЯ АВТОРОМ В ОСНОВНОМ НА ПРИМЕРАХ ИЗ РУССКОЙ ЛИТЕРАТУРЫ. РОЛИ ГУВЕРНЕРОВ В ЖИЗНИ СЕМЬИ Л.Н. ТОЛСТОГО ПОСВЯЩЕНА СТАТЬЯ А. ПОЛОСИНОЙ, В КОТОРОЙ ДЕМОНСТРИРУЕТСЯ, КАК РЕАЛЬНЫЕ УЧИТЕЛЯ, РАБОТАВШИЕ В СЕМЬЕ ПИСАТЕЛЯ, СТАНОВИЛИСЬ ПРОТОТИПАМИ ЛИТЕРАТУРНЫХ ПЕРСОНАЖЕЙ, А ТАКЖЕ ДЕЛАЕТСЯ ПОПЫТКА ОПРЕДЕЛИТЬ ВЛИЯНИЕ ФИГУРЫ ФРАНЦУЗСКОГО ГУВЕРНЕРА НА ЛИЧНОСТЬ САМОГО Л.Н. ТОЛСТОГО.

6 ТРЕТИЙ РАЗДЕЛ КНИГИ ПОСВЯЩЕН ОТДЕЛЬНЫМ ИСТОРИЧЕСКИМ ПЕРСОНАЖАМ. ГЛАВА, НАПИСАННАЯ М.БЁМ, РАСКРЫВАЕТ ИСТОРИЮ ВЗАИМООТНОШЕНИЙ БУДУЩЕГО ПРУССКОГО КОРОЛЯ ФРИДРИХА II С БЛЕСТЯЩИМ ИНТЕЛЛЕКТУАЛОМ ЖАКОМ ДЕ ЖАНДЕНОМ, КОТОРЫЙ НА ПРОТЯЖЕНИИ МНОГИХ ЛЕТ БЫЛ УЧИТЕЛЕМ ПРУССКОГО ПРИНЦА. ФРАНЦУЗСКИЙ ВОСПИТАТЕЛЬ ОКАЗАЛ ЗНАЧИТЕЛЬНОЕ ВЛИЯНИЕ НА ФОРМИРОВАНИЕ МИРОВОЗЗРЕНИЯ ФРИДРИХА. НА ОСНОВАНИИ УЧЕБНЫХ ПЛАНОВ ДЕ ЖАНДЕНА АВТОР ПЫТАЕТСЯ ПРОСЛЕДИТЬ ВЛИЯНИЕ ГУВЕРНЕРА НА СИСТЕМУ ПРЕПОДАВАНИЯ В ПРУССИИ В ЦЕЛОМ [С. 244]. О ЛИЧНОСТИ, НАВЕРНОЕ, САМОГО ИЗВЕСТНОГО ГУВЕРНЕРА, СЛУЖИВШЕГО В РОССИИ, ВОСПИТАТЕЛЯ БУДУЩЕГО ИМПЕРАТОРА АЛЕКСАНДРА І, - Ф.Ц. ЛАГАРПА - НАПИСАЛА М.П. РЕЙ. АВТОР РАССМАТРИВАЕТ ОСНОВНЫЕ ПЕДАГОГИЧЕСКИЕ ПРИНЦИПЫ И МЕТОДЫ ШВЕЙЦАРЦА И ИХ ПОЗДНЕЙШЕЕ ОТРАЖЕНИЕ В ДЕЯНИЯХ ЦАРЯ. Н. ВОЩИНСКАЯ РАССКАЗЫВАЕТ О СУДЬБЕ ШАРЛЯ МАССОНА, БЫВШЕГО ВОСПИТАТЕЛЕМ ДЕТЕЙ ГЕНЕРАЛА ГРАФА Н.И. САЛТЫКОВА, 
А ТАКЖЕ ВЕЛИКИХ КНЯЗЕЙ АЛЕКСАНДРА И КОНСТАНТИНА. ЭТОТ ПРИМЕР ЕЩЕ РАЗ ПОКАЗЫВАЕТ, ЧТО АРИСТОКРАТИЯ РОССИИ ЧАСТО ВПОЛНЕ УДАЧНО ОТБИРАЛА ПРЕПОДАВАТЕЛЕЙ ДЛЯ СВОИХ ДЕТЕЙ. ВПОЛНЕ КОМПЕТЕНТНЫМ ПРЕПОДАВАТЕЛЕМ МОЖНО ПРИЗНАТЬ И ЖИЛЬБЕРА РОММА, ПРЕПОДАВАВШЕГО В СЕМЬЕ ГРАФА АЛЕКСАНДРА СТРОГАНОВА, О КОТОРОМ НАПИСАЛ А. ЧУДИНОВ. В ГЛАВЕ, НАПИСАННОЙ В. СОМОВЫМ, РЕЧЬ ИДЕТ ОБ ИЗВЕСТНОМ ДЕЯТЕЛЕ ЭПОХИ ПРОСВЕЩЕНИЯ Н.Г. ЛЕКЛЕРКЕ, КОТОРЫЙ, В ОТЛИЧИЕ ОТ МНОГИХ УПОМЯНУТЫХ В ЭТОЙ КНИГЕ ПЕРСОНАЖЕЙ, НЕ БЫЛ ГУВЕРНЕРОМ, А ЗАНИМАЛ РАЗЛИЧНЫЕ ПОСТЫ В ГОСУДАРСТВЕННЫХ ОБРАЗОВАТЕЛЬНЫХ УЧРЕЖДЕНИЯХ (СУХОПУТНОМ ШЛЯХЕТНОМ КАДЕТСКОМ КОРПУСЕ И ИМПЕРАТОРСКОЙ АКАДЕМИИ ХУДОЖЕСТВ) И НА ЭТИХ ПОСТАХ ОКАЗЫВАЛ ЗАМЕТНОЕ ВЛИЯНИЕ НА ОБРАЗОВАТЕЛЬНУЮ ПОЛИТИКУ ЭТИХ ЗАВЕДЕНИЙ. КРОМЕ ТОГО, БУДУЧИ ЗНАКОМ С ПЕРВЫМИ ЛИЦАМИ ГОСУДАРСТВА И ИМЕЯ ВОЗМОЖНОСТЬ ОЗНАКОМИТЬ ИХ СО СВОИМИ ТРАКТАТАМИ О ВОСПИТАНИИ, ЛЕКЛЕРК ОКАЗЫВАЛ КОСВЕННОЕ ВЛИЯНИЕ НА ОБРАЗОВАТЕЛЬНУЮ СИСТЕМУ РОССИЙСКОЙ ИМПЕРИИ В ЦЕЛОМ. А. БАНДЕЛЬЕ И В. РЖЕУЦКИЙ В СВОЕЙ ГЛАВЕ ДАЮТ КРАТКУЮ ХАРАКТЕРИСТИКУ СЕТИ ФРАНКОЯЗЫЧНЫХ УЧИТЕЛЕЙ ИЗ ШВЕЙЦАРИИ, РАБОТАВШИХ В РОССИИ В XVIII-XIX ВВ., БОЛЕЕ ПОДРОБНО ОСТАНАВЛИВАЯСЬ НА ОПИСАНИИ БИОГРАФИИ НИКОЛЯ ФОРНЕРО, ДО СИХ ПОР МАЛО ИЗВЕСТНОЙ ИССЛЕДОВАТЕЛЯМ. КРОМЕ ТОГО, АВТОРЫ АНАЛИЗИРУЮТ СОЧИНЕНИЕ ФОРНЕРО О РОССИИ, КОТОРОЕ ХОТЯ И НЕ ПОЛУЧИЛО В ТО ВРЕМЯ БОЛЬШОЙ ИЗВЕСТНОСТИ, ПРЕДСТАВЛЯЕТ ИНТЕРЕС, ПОСКОЛЬКУ ДЕМОНСТРИРУЕТ НЕ ТОЛЬКО ОБРАЗ РОССИИ, СФОРМИРОВАВШИЙСЯ В СОЗНАНИИ ИНОСТРАННОГО НАБЛЮДАТЕЛЯ, НО И ПОЗВОЛЯЕТ РАСШИРИТЬ НАШИ ЗНАНИЯ ОБ ИНОСТРАННЫХ ВОСПИТАТЕЛЯХ, РАБОТАВШИХ В РОССИИ. Ш. ГЁЦ ПОСВЯТИЛ СВОЕ ИССЛЕДОВАНИЕ СУДЬБЕ ДАВИДА ДЕ БУДРИ, БРАТА РЕВОЛЮЦИОНЕРА МАРАТА, СЛУЖИВШЕГО ВОСПИТАТЕЛЕМ В СЕМЬЕ САЛТЫКОВЫХ, А ЗАТЕМ ПРОДОЛЖИВШЕГО КАРЬЕРУ В ГОСУДАРСТВЕННЫХ УЧЕБНЫХ ЗАВЕДЕНИЯХ : ИНСТИТУТЕ СВ. ЕКАТЕРИНЫ АЛЕКСАНДРИЙСКОЙ, САНКТ-ПЕТЕРБУРГСКОЙ ГУБЕРНСКОЙ ГИМНАЗИИ И ЦАРСКОСЕЛЬСКОМ ЛИЦЕЕ. Н. КРЯЖЕВА РАССКАЗАЛА О Ж.Б. МОДРЮ, АВТОРЕ ПЕРВОЙ ГРАММАТИКИ РУССКОГО ЯЗЫКА, ОПУБЛИКОВАННОЙ ВО ФРАНЦИИ. В ГЛАВЕ КРАТКО РАССМАТРИВАЮТСЯ ПРЕДСТАВЛЕНИЯ МОДРЮ О СИСТЕМЕ ПРЕПОДАВАНИЯ ГРАММАТИКИ РАЗНЫХ ЯЗЫКОВ И МЕТОДИКЕ ОБУЧЕНИЯ ЧТЕНИЮ, КОТОРЫЕ БЫЛИ ВО МНОГОМ НОВАТОРСКИМИ ДЛЯ СВОЕГО ВРЕМЕНИ.

7 ПРЕДСТАВЛЕННЫЙ ТРУД НАГЛЯДНО ДЕМОНСТРИРУЕТ, ЧТО ЛИТЕРАТОРЫ МНОГИХ СТРАН, ИЗОБРАЖАВШИЕ ФРАНЦУЗСКИХ ГУВЕРНЕРОВ МАЛОКОМПЕТЕНТНЫМИ В ОБРАЗОВАНИИ ЛЮДЬМИ, БЫЛИ ДАЛЕКО НЕ ВСЕГДА ПРАВЫ. НАРЯДУ СО СЛУЧАЙНЫМИ ЛИЦАМИ И АВАНТЮРИСТАМИ, СРЕДИ ЧАСТНЫХ ПРЕПОДАВАТЕЛЕЙ БЫЛО НЕМАЛО ХОРОШО ОБРАЗОВАННЫХ ЛЮДЕЙ, УЧЕНЫХ И ПЕДАГОГОВ. ИМЕННО ТАКИЕ ФИГУРЫ ОКАЗАЛИ ЗАМЕТНОЕ ВЛИЯНИЕ НА ФОРМИРОВАНИЕ НАЦИОНАЛЬНЫХ СИСТЕМ ОБРАЗОВАНИЯ В РАЗЛИЧНЫХ СТРАНАХ ЕВРОПЫ. КРОМЕ ТОГО, МАТЕРИАЛЫ ИЗДАНИЯ ПОЗВОЛЯЮТ ПРОСЛЕДИТЬ ПРОЦЕСС ПОСТЕПЕННОГО ВЫТЕСНЕНИЯ ЧАСТНЫХ ПРЕПОДАВАТЕЛЕЙ И СОЗДАНИЯ ГОСУДАРСТВЕННЫХ СИСТЕМ ОБРАЗОВАНИЯ, ЧТО ОСОБЕННО ЗАМЕТНО НА МАТЕРИАЛАХ ПО РОССИЙСКОЙ ИСТОРИИ ${ }^{2}$. 


\section{NOTES}

1. Anne Mézin, Vladislav Rjéoutski, éds., Les Français en Russie au siècle des Lumières. Dictionnaire des Français, Suisses, Wallons et autres francophones en Russie de Pierre le Grand à Paul I ${ }^{\text {er }}$, Centre international d'étude du XVIII ${ }^{\mathrm{e}}$ siècle, 2011, 2 vol. , 1424 p.

2. The review was supported by Russian scientific fund, project N 14-18-01116 Social mechanisms of a mass protest movement during the French Revolution and the Napoleonic Empire.

\section{AUTEURS}

\section{NIKOLAJ PROMYSLOV}

НАУЧНЫЙ СОТРУДНИК ИНСТИТУТА ВСЕОБЩЕЙ ИСТОРИИ РАН 